\title{
OLIGOMERIZAÇÃO DO GLICEROL UTILIZANDO CATALISADORES BÁSICOS HOMOGÊNEOS: NaOH E $\mathrm{CaO}$
}

\author{
F. J. S. BARROS ${ }^{1}$, F. L. MARQUES ${ }^{1}$, R. S. VIEIRA ${ }^{1}$, e F. M. T. de LUNA ${ }^{1}$ \\ ${ }^{1}$ Universidade Federal do Ceará, Departamento de Engenharia Química \\ E-mail para contato: fernando_barros17@yahoo.com.br
}

\begin{abstract}
RESUMO - Este trabalho objetiva avaliar catalisadores básicos homogêneos na reação de oligomerização do glicerol. As reações foram processadas em reator batelada, utilizando-se glicerol P.A e $\mathrm{NaOH}$ ou $\mathrm{CaO}$ como catalisadores. As reações ocorreram com fluxo de nitrogênio, a $245^{\circ} \mathrm{C}$ e duração de 6 horas. Para fins comparativos, também realizou-se um teste sem catalisador. A caracterização via infravermelho evidenciou a redução acentuada do pico característico do grupo $\mathrm{OH}$, para a reação catalisada por $\mathrm{NaOH}$, bem como a possível formação de acroleína. Os ensaios reológicos mostraram que o produto catalisado por $\mathrm{CaO}$ possui menor viscosidade em relação ao glicerol, enquanto que o catalisado pelo $\mathrm{NaOH}$ apresentou viscosidade similar ao mesmo. A partir da viscosidade intrínseca dos compostos verificou-se que o composto sintetizado com $\mathrm{CaO}$ apresentou maior massa molar viscosimétrica, cerca de 15 vezes a massa do glicerol.
\end{abstract}

\section{INTRODUÇÃO}

O glicerol é o principal subproduto da reação de transesterificação para a produção do biodiesel. Para cada dez litros de biodiesel, cerca de um litro de glicerina é produzido, o que equivale a um acúmulo mundial em larga escala desse subproduto (PAGLIARO; ROSSI, 2008).

De acordo com Umpierre e Machado (2013) este excedente de glicerol limita definitivamente sua estocagem em longo prazo e sua degradação microbiana em taxas suficientemente altas. Portanto, o glicerol é um produto cada vez mais abundante, que deve ser convertido industrialmente através de novas rotas catalíticas e de menor impacto ambiental em comparação com as rotas tradicionais.

A indústria de tintas foi a primeira a intensificar o consumo da glicerina como material para a produção de resinas alquídicas ou como reticulante, disputando a aplicação com o conhecido pentaeritritol sintético. O uso nas tintas comporta de $20 \%$ a $30 \%$ da glicerina loira, bidestilada, produzida no Brasil (FAIRBANKS, 2009).

Dentre as possíveis reações para a valorização do glicerol, encontra-se as reações de polimerização, com notado destaque para a obtenção de di e triglicerol. Segundo Behr et 
al (2008) estes são éteres com grande potencial de utilização na síntese de materiais poliméricos. Normalmente, a formação de oligômeros de glicerol (diglicerol e triglicerol) ocorre via reação entre o grupamento hidroxila primária, conduzindo a formação de oligômeros com cadeia linear.

Este trabalho visa avaliar o desempenho de catalisadores básicos homogêneos na reação de oligomerização do glicerol sem a presença de solvente. As amostras foram analisadas via Infravermelho e tiveram suas viscosidades dinâmica e intrínseca determinadas, também foi obtida a massa molecular viscosimétrica.

\section{2 .MATERIAL E MÉTODOS}

Utilizaram-se nas reações Glicerol P.A (Vetec), e como catalisadores $\mathrm{NaOH}$ (Vetec) e $\mathrm{CaO}$ (Vetec), também foi realizado um teste sem a presença de catalisador. As reações ocorreram em um reator Parr, a $245^{\circ} \mathrm{C}$, com atmosfera inerte provocada pela passagem de nitrogênio, por um período de 6 horas.

\subsection{Viscosidade Intrínseca}

A determinação da viscosidade intrínseca, foi realizada a $40 \pm 0,1^{\circ} \mathrm{C}$, utilizandose um viscosímetro capilar Cannon - Fenske 50, colocado em um banho termostatizado e a partir dela foi calculada a massa molecular média. Pelo método de extrapolação gráfica à diluição infinita, a determinação experimental foi feita pela medição do tempo de escoamento de cinco soluções poliméricas diluídas $(0,01 ; 0,03,0,05 ; 0,07 ; 0,09)$, a partir de uma solução mãe de $0,20 \%(\mathrm{~m} / \mathrm{v})$ da amostra em água. Os testes foram realizados em duplicata.

Os cálculos da viscosidade específica e reduzida para cada solução e a determinação da viscosidade intrínseca [ $\eta]$, foram baseados nas seguintes expressões.

Viscosidade relativa: $\eta_{r e l}=\eta_{s o l} / \eta_{o}=t_{s o l} / t_{o}$

onde $\eta_{0}$ é a viscosidade do solvente (água ) sem polímero e $\rho_{o}$ e $\rho_{\text {sol }}$ são as densidades. Nesta relação, assume-se que as densidades da solução e do solvente são iguais, haja visto tratar-se de uma solução extremamente diluída.

Viscosidade específica: $\eta_{s p}=\left(\eta_{s o l}-\eta_{o}\right) / \eta_{o}=\eta_{r e l-1}$

Viscosidade reduzida: $\eta_{r e d}=\eta_{s p} / c$

, onde c é a concentração em $\mathrm{g} \mathrm{mL}^{-1}$

Viscosidade intrínseca: $[\eta]=\lim (c \rightarrow 0) \eta_{s p} / c$

A relação entre viscosidade intrínseca e massa molar é dada pela correlação de Staudinger-Mark-Houwink apresentada na Equação 5:

Em que:

$$
[\eta]=K M_{v}{ }^{a}
$$

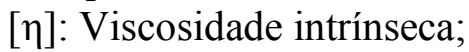

$\mathrm{K}$ : constante referente ao polímero;

a : constante referente ao polímero;

Mv: Massa molecular viscosimétrica.

Na ausência dos parâmetros da correlação de Mark - Houwink - Staudinger, foram utilizados valores para $\mathrm{K}$ a de um polímero que interagisse de forma similar com o mesmo solvente do poliglicerol, neste caso o Poliálcool Vinílico (PVA), que apresenta $\mathrm{K}=0,0453 \mathrm{~mL} / \mathrm{g}$ e $\mathrm{a}=0,64$. 


\subsection{Viscosidade Dinâmica}

Para a determinação da viscosidade dos produtos obtidos foi utilizado um viscosímetro da marca Brookfield (Modelo DV-III ULTRA). O instrumento é equipado com cilindros de diâmetros diferentes (spindles), selecionados conforme a viscosidade do fluido. Para este trabalho foi utilizado o spindle de referência 34, um cilindro de diâmetro externo de $9,39 \mathrm{~mm}$ e $24,23 \mathrm{~cm}$ de comprimento.

Para a realização das análises reológicas, foi utilizado, para cada medida, $11 \mathrm{~mL}$ de amostra, submetida a estabilização prévia por 15 minutos. O equipamento mede a viscosidade de fluidos através da taxa de cisalhamento. Além dos poligliceróis também foi analisado o glicerol utilizado como reagente.

\subsection{Espectroscopia Vibracional na Região do Infravermelho com Transformadas de Fourier (FTIR)}

Para essa análise foi utilizado o equipamento IR-PRESTIGE 21 SHIMADZU FTIR com células de $\mathrm{KBr}$. A faixa de varredura usada nas análises foi de 400 a 4000 $\mathrm{cm}^{-1}$, com resolução espectral de $8,0 \mathrm{~cm}^{-1}$. Amostras líquidas foram preparadas por disposição de alíquota no próprio suporte de $\mathrm{KBr}$.

\section{RESULTADOS E DISCUSSÃO}

\subsection{Viscosidade Intrínseca}

A Figura 1 mostra os gráficos obtidos no teste reológico e utilizados para o cálculo dos valores de viscosidade intrínseca e massa molecular viscosimétrica (Mv), apresentados na Tabela 1, obtidos pelo método descrito anteriormente.

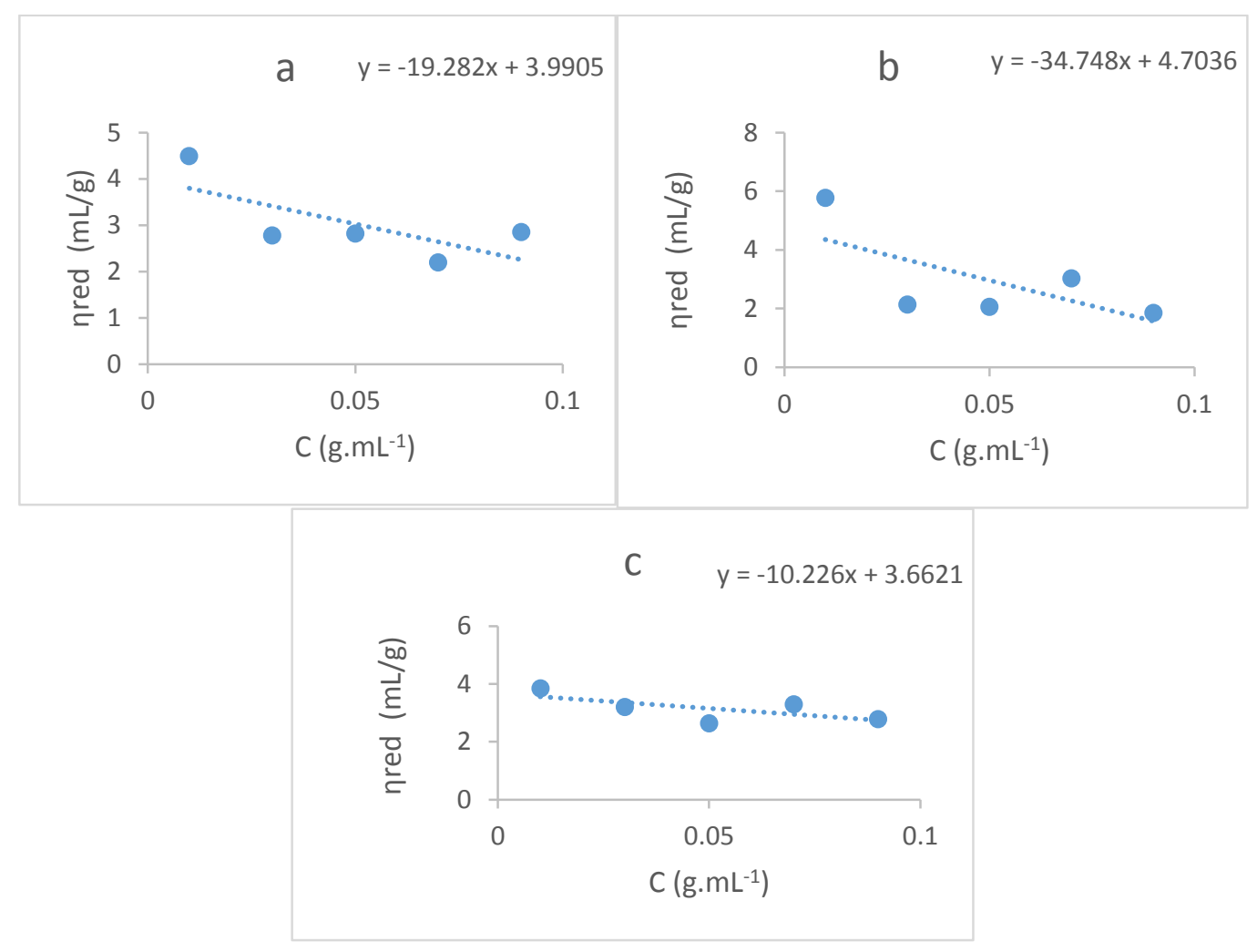

Figura 1 - Curvas Viscosidade Reduzida x Concentração: a) Sem

Catalisador $+6 \mathrm{~h}+245^{\circ} \mathrm{C}$ com fluxo de $\mathrm{N}_{2}$ b) Catalisador por $\mathrm{CaO}+6 \mathrm{~h}+245^{\circ} \mathrm{C}$ com fluxo de $\mathrm{N}_{2}$ c) Catalisado por $\mathrm{NaOH}+6 \mathrm{~h}+245^{\circ} \mathrm{C}$ com fluxo de $\mathrm{N}_{2}$. 
Tabela 1 - Viscosidade Intrínseca e Massa molar média

\begin{tabular}{|c|c|c|c|}
\hline $\begin{array}{c}\text { Catalisador } \\
\text { utilizado }\end{array}$ & $\begin{array}{c}\text { Percentual de } \\
\text { Catalisador }(\%)\end{array}$ & $\begin{array}{c}\text { Viscosidade } \\
\text { Intrínseca }(\mathrm{mL} / \mathrm{g})\end{array}$ & $\mathrm{Mv}\left(\mathrm{g} \cdot \mathrm{mol}^{-1}\right)$ \\
\hline $\mathrm{NaOH}$ & 2 & 3.66 & 956.48 \\
\hline $\mathrm{CaO}$ & 2 & 4.70 & 1414.23 \\
\hline Sem catalisador & - & 3.99 & 1093.83 \\
\hline
\end{tabular}

$\mathrm{O}$ produto obtido via catálise com $\mathrm{CaO}$ apresentou elevada massa molecular viscosimétrica, e maior grau de polimerização que os demais, cerca de 15 vezes a massa do glicerol, face o composto catalisado pelo $\mathrm{NaOH}$ (10 vezes) e o teste sem catalisador (12 vezes). Este resultado aponta para uma maior eficiência do $\mathrm{CaO}$ na oligomerização do glicerol.

\subsection{Viscosidade Dinâmica}

A tabela 2 mostra os resultados obtidos nos ensaios de viscosidade dinâmica.

Tabela 2- Viscosidade Dinâmica dos produtos

\begin{tabular}{|c|c|c|}
\hline Amostra & $\begin{array}{c}\text { Percentual de } \\
\text { Catalisador }(\%)\end{array}$ & Viscosidade Dinâmica (cP) \\
\hline Glicerol P.A & - & 278.9 \\
\hline Produto catalisador por $\mathrm{NaOH}$ & 2 & 300.7 \\
\hline Produto catalisador por $\mathrm{CaO}$ & 2 & 86.83 \\
\hline Produto Sem catalisador & - & 76.18 \\
\hline
\end{tabular}

Observa-se que nos testes com $\mathrm{CaO}$ e sem catalisador houve significativa redução da viscosidade dos produtos em relação ao glicerol, enquanto que no teste com $\mathrm{NaOH}$ o resultado foi semelhante a do reagente. A redução nas viscosidades é esperada haja visto que a medida que a reação de polimerização avança, as cadeias carbônicas dos éteres formados se tornam maiores e mais complexas, devido principalmente a formação de ramificações e algumas ligações entre cadeias paralelas de oligômeros e/ou polímeros, o que modifica suas propriedades, tais como a viscosidade.

Segundo Mota et al., 2009, a eterificação do glicerol possui a característica de produzir composto que apresentam menor viscosidade, fato que contribui bastante na miscibilidade com outras substâncias.

\subsection{Espectroscopia Vibracional na Região do Infravermelho com Transformadas de Fourier (FTIR)}

A figura 2 apresenta os espectros obtidos via FTIR. 


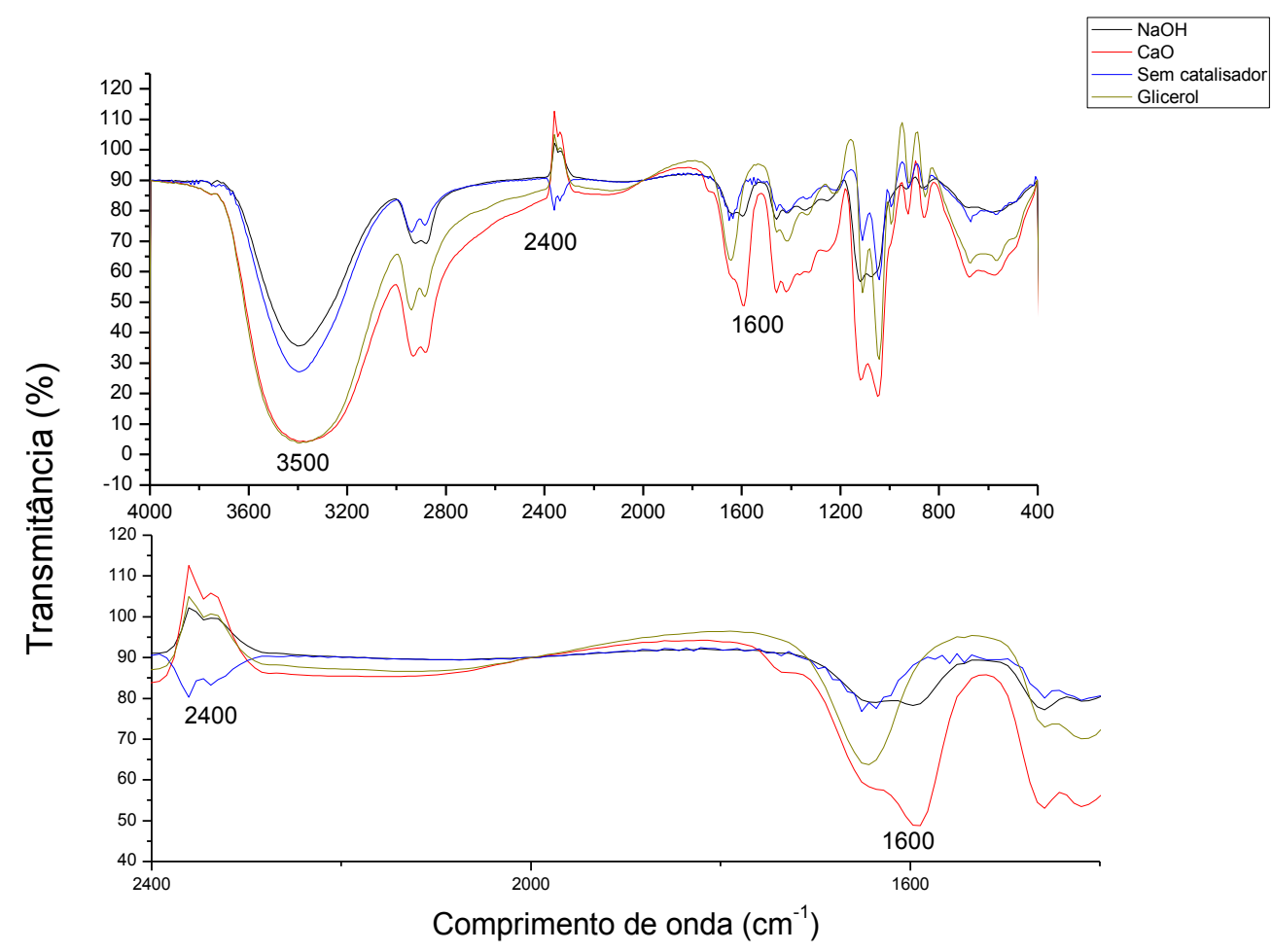

Figura 2 - Espectro infravermelho do glicerol puro, dos produtos obtidos via catalise com $\mathrm{NaOH}, \mathrm{CaO}$ e sem catalisador

Os espectros de infravermelho dos poligliceróis formados apresentaram uma banda na região de alto número de onda $\left(3500 \mathrm{~cm}^{-1}\right)$ característica da deformação axial simétrica e assimétrica do grupo hidroxila $\mathrm{OH}$. A reação catalisada por $\mathrm{NaOH}$ apresentou maior redução desta banda, indicativo de um maior desprendimento das hidroxilas na formação dos oligômeros. Esta técnica possibilitou, também, observar o aparecimento de picos em torno de $1600 \mathrm{~cm}^{-1}$, região característica da molécula de acroleína $\mathrm{v}(-\mathrm{C}=\mathrm{C}-)$. $\mathrm{O}$ surgimento de picos nas regiões de acetonas foi observado em $1644 \mathrm{~cm}^{-1}$ e $2400 \mathrm{~cm}^{-1}$.

\section{CONCLUSÃO}

A partir das técnicas utilizadas foi possível verificar a oligomerização do glicerol e quantificar a massa molecular dos compostos. Destacam-se os resultados obtidos com $\mathrm{CaO}$, que apresentou um maior grau de polimerização, cerca de 15 vezes a massa do glicerol. Os testes de viscosidade dinâmica apontaram para a diminuição da viscosidade dos poligliceróis em relação ao glicerol, em concordância com a literatura. As análises de infravermelho evidenciaram a redução dos grupamentos hidroxila, e consequentemente a formação de oligômeros, bem como a presença de acroleína e acetonas.

\section{AGRADECIMENTOS}

Os autores gostariam de agradecer à Capes e à Funcap pelas bolsas concedidas. 


\section{REFERÊNCIAS}

BEHR, A.; EILTING, J.; IRAWADI, K.; LESCHINSKI, J.; LINDNER, F.Green Chem. ,2008.

FAIRBANKS, M. Crescimento do biodiesel provoca inundação no mercado de glicerina, incentivando a descobrir novas aplicações. Química e Derivados, Ed. nº 487, p. 1-12, 2009.

KNOTHE, G.; GERPEN, J. V.; KRAHL, J.; RAMOS, L. P. Manual do Biodiesel. São Paulo: Editora Blucher, 2006.

MOTA, C. J. A; SILVA, C. X. A. da; GONÇALVES, V.L. C; Gliceroquímica: novos produtos e processos a partir da glicerina de produção de biodiesel. Química Nova. v. 32. no 3. p. 639-648,2009.

PAGLIARO, M.; ROSSI, M. Future of Glycerol: New Usages for a Versatile Raw Material. Green Chemistry Series. Royal Society of Chemistry, 1st edition, p.104, 2008.

UMPIERRE, A. P.; MACHADO, F. Gliceroquímica e Valorização do Glicerol. Rev. Virtual Quim., p. 106-116. 2013. Disponível em <http://www.uff.br/rvq>. Acesso em 20 de Março de 2014. 